\title{
Commentary on "The Happy Xylophone: Acoustics Affordances Restrict An Emotional Palate" by Michael Schutz, David Huron, Kristopher Keeton, \& Greg Loewer
}

\author{
ZOHAR EITAN \\ Tel Aviv University
}

\begin{abstract}
In this commentary, I raise several issues of method and presentation and suggest a number of follow-up experiments associated with some of these issues. Broad suggestions are also made (or rather preached): the need to deal empirically with musical emotions subtler than the oft-investigated basic emotions, and the role that interactions between musical variables may play in shaping subtle musical expression, as exemplified by some well-known xylophone soli from the orchestral repertory.
\end{abstract}

Submitted 2008 August 31; accepted 2008 September 2.

KEYWORDS: timbre, acoustic, valence, affect, xylophone

NOMINALLY, "The Happy Xylophone" examines how a bundle of three acoustic features - pitch register, "brightness" of timbre, and articulation rate - correlates with the structural musical feature of mode (major vs. minor). Low pitch register, dull timbre, and legato articulation have been empirically associated with the expression of sad emotion in both speech and music. In music, the minor mode is also a strong (though not a necessary or sufficient) predictor of expressed or perceived sadness. Of the mallet instruments, the modern concert xylophone is devoid of the above "sad" acoustical correlates, as it is restricted to a high pitch range, sharp timbre, and very short note durations (which, the authors suggest, practically rule out long IOI); in contrast, the marimba is less constrained in these regards, featuring a wider (and lower) pitch range, darker sound, and more sustained notes. Hence, the article hypothesizes that the minor mode associated with sad expression - would be generally avoided in solo music for the xylophone, but widely featured in marimba music. Corroborating this hypothesis through a comparison of current American concert repertories of the two instruments, the authors conclude that "Essentially, the acoustic structure of the xylophone has type-cast it as a happy instrument."

\section{ISSUES OF METHOD AND PRESENTATION}

\section{What does this sample represent?}

While investigating current American concert repertory involving mallet instruments, the study implies wider, perhaps universal relationships between psychoacoustic constraints (high register, short duration, and sharp timbre) and musical affect, as conveyed by musical mode. Yet, the sample analyzed represents a relatively small and recent musical sub-culture, heavily influenced by a few noted performers and performer-composers (e.g., Leigh Howard Stevens, George Hamilton Green, Joe Green) and by specifically American trends, such as the popularity of Ragtime compositions for xylophone in the early $20^{\text {th }}$ century and beyond. Indeed, only six composers are represented in the xylophone sample, four of which are Americans. To what degree, then, does the sample represent more than a small, particular musical subculture? Would it apply as well even to the contemporary European or Japanese concert repertory for the same instruments?

Notably, the orchestral repertory provides some well-known examples of prominent xylophone parts whose emotional connotations, as implied by the pieces' titles, texts, or programs, are not exactly "happy" (though, importantly, neither are they "sad."). Rather, these pieces suggest emotions and expressive stances such as lamentation and anguish (Shostakovich, Symphony no. 14, $5^{\text {th }}$ movement; Symphony no. 5, $3^{\text {rd }}$ movement), the macabre (Saint Saens, Danse Macabre), aggressiveness (Prokoviev, Ala et Lolly, Khachaturian's Sabre Dance) or heavy irony and sarcasm (Shostakovich, Polka from the Golden Age). While such small "sample" does not, of course, refute the authors' statistics (for one thing, 
most of the above examples apply neither minor nor major modes), it does point out the need to widen their population base. In short, one cannot really know to what degree the results presented here reflect general musical tendencies or rather embody the particular traits, influences and history of a contemporary American musical sub-culture.

\section{Disentangling a bundle}

Relating a bundle of three independent variables (which, in the present case, cannot be dissociated from one another) to a dependent variable raises an obvious issue: to what degree does each of these variables (pitch register, timbre "lightness", and rate of articulation) contribute to the results reported (scarcity of minor mode compositions, and presumably, expressed happiness)? The current design does not allow for an examination of the individual effects and interactions of the three variables examined. We cannot infer from the present study whether all three variables actually contribute to the scarcity of the minor mode in the xylophone repertory, nor the relative contribution of each.

While the three acoustic features associated with the xylophone have been related in previous empirical studies to positively-valenced emotions like happiness and joy, they have also been significantly associated with the negative emotions of anger and fear (though not to sadness; see the summarizing table 10.2 in Gabrielsson and Lindström, 2001, pp. 235-239). Importantly, all three features correlate not only with positive valence, but also with high activity and arousal (Gabrielsson \& Juslin, 2003). When interacting with variables that tend to convey negative valence, their arousal potential may override their tendency to express positive valence, resulting in negative yet active emotions like anger. That the composers included in the present sample have chosen not to employ such interactions may attest not to any obligatory, "natural" association of xylophones with happiness, but rather (as suggested above) to particular trends of musical style and culture. Alternatively, when all three acoustic variables conjoin, as in a xylophone sound, their combined effect may all but impose positive valence, as the authors suggest. Given the current design, we simply cannot know which of these two options prevails.

\section{Studying emotion directly}

As noted, the study suggests that the xylophone's acoustic constraints almost necessarily induce a happy expression, basing this conclusion on the avoidance of minor mode compositions in a xylophone repertory. Indeed, as the authors point out, the minor mode has been associated, more decisively than any other musical feature, with sadness. Thus, the above reservations notwithstanding, the relationship revealed here between acoustic features and musically-expressed emotional valence is important and intriguing. Yet, the assumed "happiness" of xylophone music, as compared to the marimba, may be examined more directly, for instance through listeners' valence ratings -- continuous or global -- of the music (see Gabrielsson \& Lindström, 2001, for a survey of relevant methodologies). In other words: why use an indirect (though relatively reliable) measure of emotion, when direct measures are readily available?

\section{Musical structure and acoustic features}

The most important contribution of this article, however, perhaps lies not in the study of emotional expression in music per se. Rather, demonstrating an effect of acoustic constraints on the use of an important structural feature in music (such as mode) is important in and of itself. "The Happy Xylophone," regardless of the instrument's presumed emotional stance, thus extends an approach previously used by Huron (2001) in investigating the effects of perceptual constraints on the rules of tonal voice-leading, demonstrating in a different way how the use of high-level, music-specific structures may be constrained by low-level psychoacoustic constraints.

\section{Invoking speech}

The study examines, within a musical repertory, features that have all been shown to relate to emotion in music. Pitch register, note duration, and brightness of timbre have all been associated with valence in a large number of studies of music perception and performance, some of which (e.g., Hevner, 1935, 1937; see Gabrielsson \& Lindström, 2001, for a survey) have preceded comparable studies of speech by decades. Though the relationship of music with speech intonation or prosody evidently raises intriguing and important issues, this article does not deal with these issues in any direct way. Hence, presenting musicspeech relationships as central to its arguments (as the abstract and opening paragraphs do) is somewhat confusing. In short, since the study's hypothesis can be squarely based upon empirical findings regarding 
music, the issue of speech-music relationships, though extremely important generally, is rather marginal in the context of this article.

\section{SOME FOLLOW-UP EMPIRICAL INVESTIGATIONS}

The clichés stating that the best compliment for an empirical study are the questions and hypotheses it raises, and the follow-up studies those may generate, rightly apply to "The Happy Xylophone." Here, very briefly, are some suggestions for such follow-ups.

\section{Extending the examined population}

Even given the original object of this study - comparing the frequency of minor and major mode pieces in xylophone and marimba repertories - examining repertories beyond the limited place (United States), time (now) and genre (solo pieces for mallet instruments) applied in "The Happy Xylophone" may be worth while. Similar examinations of European solo repertories, and of xylophone and marimba solos in orchestral repertories, are probable options, as would be an investigation of earlier concert programs. Such investigations would clarify whether the relationship of mode with the xylophone's bundle of acoustic properties can be generalized beyond the American musical and cultural sphere, thereby testing the external validity of the article's bold hypothesis.

\section{Disentangling the feature bundle}

Trying to assess the individual contribution and interaction of the three acoustic parameters focused upon in this article would suggest different research paradigms, including statistical examination of musical repertories as well as cognitive experiments.

Repertory studies can examine whether mode (major vs. minor) is significantly related to tempo (or IOI), pitch register, or timbre (sharp vs. dull) in tonal repertories, while trying to disentangle (and control for) possible correlations of the three acoustic variables with each other (e.g., higher pitch and faster tempo). For instance, is the minor mode more frequent in solo viola music, as compared to that of the violin? Is the sharper sul ponticello (near the bridge) sound in string instruments, strengthening highfrequency partials, more often associated with the major mode than sul tasto or a normal sound? Are major mode compositions (or movements) generally faster than minor mode ones (an intuitively plausible association which, as far as I know, has not been widely investigated)?

Cognitive experiments can be conducted to investigate whether listeners actually associate these acoustic variables with the major-minor polarity. For instance, would aesthetic ratings (e.g., liking, expressiveness, interest) of a slow minor melody be higher than those of the same melody played faster? Would lowering overall pitch register enhance the aesthetic ratings or preference for a minor phrase? Would muting upper partials have a comparable effect? Indeed, how would playing a minor melody alternatively on the xylophone or the marimba, while controlling for pitch register (as the two instruments' ranges partially overlap) and tempo affect aesthetic ratings or preferences? Perhaps most importantly, how would different, systematically controlled combinations of the three acoustic variables affect listeners' preferences for major or minor modality?

\section{A NOTE ON INCONGRUENCE AND COMPLEX EXPRESSIVE STANCES}

The $5^{\text {th }}$ movement of Shostakovich's $14^{\text {th }}$ symphony, op. 135, sets an Apollinaire text, Les Attentives no. 1, in which a woman in utter despair mourns the impending death of her soldier lover on the trenches (see Figure 1). Throughout the movement, a solo xylophone repeats a single theme, which exemplifies many of the features related in "The Happy Xylophone" (and elsewhere in the empirical literature) to sentiments of joy and happiness: a high pitch level, a sharp timbre (here emphasized by a forte dynamics), a fast tempo, as well as leaps of perfect 4ths and 5ths. No major mode, though, is featured here, but a 12 tone row, continuously challenging the tonal implications of the theme's perfect melodic intervals. To this writer, the combination of a continuously shattered tonality with icons of a simple, confidently happy affect seems to annunciate the bitter despair of Apollinaire's text better than any conventionally mournful set of musical features.

While "The Happy Xylophone" deftly demonstrates how congruence of different acoustic and structural elements in music can shape a simple, straight-forward affect, Shostakovich's melody (and other examples noted at the beginning of this commentary) reveals how a composer can challenge such constraints, creating a complex, incongruent interaction of different musical variables and of their affective connotations. 


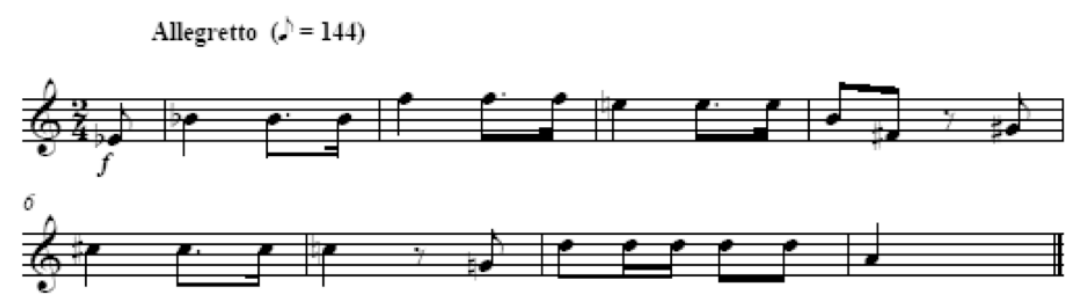

Fig. 1. Shostakovich, Symphony no. 14, V, mm. 1-8

Music, or so it is often claimed, excels at portraying such subtle, complex emotions. Empirical music research, the reviewed study included, have so far told us much of value about how musical features and their congruent use shape the expression of general expressive dimensions, such as valence or arousal, and of basic emotions like happiness, sadness or anger, stemming from them. Much less is known empirically about the expression and perception of more nuanced and complex emotions. As exemplified by Shostakovich's theme, one way towards elucidating such subtle expression may perhaps be an understanding of how various cues for emotion, both acoustic and music-specific, may sometimes conflict and interact with each other, interaction that may produce new, unexpected shades of emotion. In establishing which acoustic and structural emotional cues normatively concur with each other, "The Happy Xylophone," among other empirical studies of musical emotion, suggests what to examine when studying the denial of such concurrence, perhaps a key to understanding how expressive complexity in music works.

\section{REFERENCES}

Huron, D. (2001). Tone and Voice: A derivation of the rules of voice-leading from perceptual principles. Music Perception, Vol. 19, 1-64.

Hevner, K. (1935). The affective character of the major and minor modes in music. American Journal of Psychology, Vol. 47, 103-118.

Hevner, K. (1937). The affective value of pitch and tempo in music. American Journal of Psychology, Vol. 49, 621-630.

Gabrielsson, A., \& Juslin, P. N. (2003). Emotional expression in music. In H. H. Goldsmith, R. J. Davidson, \& K. R. Scherer (Eds.), Handbook of affective sciences. New York: Oxford University Press, pp. 503-534.

Gabrielsson, A., \& Lindström, E. (2001). The influence of musical structure on emotional expression. In P. N. Juslin, \& J. A. Sloboda (Eds.), Music and emotion: Theory and Research. New York: Oxford University Press, pp. 223-248. 